\title{
Respiratory tract infection-related healthcare utilisation in children with Down's syndrome
}

\author{
Logan Manikam ${ }^{1,2} \cdot$ Anne G. M. Schilder ${ }^{3,4} \cdot$ Monica Lakhanpaul $^{5,6} \cdot$ Peter Littlejohns $^{7} \cdot$ Emma C. Alexander $^{8}$. \\ Andrew Hayward ${ }^{1,2}$
}

Received: 18 January 2020 / Accepted: 2 March 2020 / Published online: 14 March 2020

(c) The Author(s) 2020

\begin{abstract}
Purpose Children with Down's syndrome (DS) are prone to respiratory tract infections (RTIs) due to anatomical variation, immune system immaturity and comorbidities. However, evidence on RTI-related healthcare utilisation, especially in primary care, is incomplete. In this retrospective cohort study, we use routinely collected primary and secondary care data to quantify RTI-related healthcare utilisation in children with DS and matched controls without DS.

Methods Retrospective cohort study of 992 children with DS and 4874 matched controls attending English general practices and hospitals as identified in Clinical disease research using LInked Bespoke studies and Electronic health Records (CALIBER) from 1997 to 2010. Poisson regression was used to calculate consultation, hospitalisation and prescription rates, and rate ratios. Wald test was used to compare risk of admission following consultation. The Wilcoxon rank-sum test was used to compare length of stay by RTI type and time-to-hospitalisation.

Results RTI-related healthcare utilisation is significantly higher in children with DS than in controls in terms of GP consultations (adjusted RR 1.73; 95\% CI 1.62-1.84), hospitalisations (adjusted RR 5.70; 95\% CI 4.82-6.73), and antibiotic prescribing (adjusted RR 2.34; 95\% CI 2.19-2.49). Two percent of children with DS presenting for an RTI-related GP consultation were subsequently admitted for an RTI-related hospitalisation, compared to $0.7 \%$ in controls.

Conclusions Children with DS have higher rates of GP consultations, hospitalisations and antibiotic prescribing compared to controls. This poses a significant burden on families. Further research is recommended to characterise healthcare behaviours and clinical decision-making, to optimise care for this at risk group.
\end{abstract}

Keywords Respiratory $\cdot$ Down's syndrome $\cdot$ Infections $\cdot$ Antibiotics $\cdot$ Hospitalisation $\cdot$ Community

Electronic supplementary material The online version of this article (https://doi.org/10.1007/s15010-020-01408-5) contains supplementary material, which is available to authorized users.

Logan Manikam

logan.manikam.10@alumni.ucl.ac.uk

1 UCL Institute of Epidemiology and Healthcare, University College London, 1-19 Torrington Place, London WC1E 6BT, UK

2 UCL Institute of Health Informatics Research, University College London, 222 Euston Road, London NW1 2DA, UK

3 National Institute of Health Research University College London Hospitals Biomedical Research Centre, 149 Tottenham Court Road, London W1T 7DN, UK

4 evidENT, UCL Ear Institute, University College London, 332 Grays Inn Road, London WC1X 8DA, UK
5 Population, Policy \& Practice, UCL Great Ormond Street Institute of Child Health, University College London, 30 Guilford Street, London WC1N 1EH, UK

6 Whittington Health NHS Trust, Magdala Avenue, London N19 5NF, UK

7 Centre for Implementation Science, Institute of Psychiatry, Psychology and Neurosciences, King's College London, 6 De Crespigny Park, Camberwell, London SE5 8AB, UK

8 Paediatric Liver, GI and Nutrition Centre and Mowatlabs, King's College Hospital, Denmark Hill, London SE5 9RS, UK 


\section{Introduction}

Approximately 1 in 1000 children born in England and Wales will be born with Down's syndrome (DS), or trisomy 21 . This equates to around 750 children born with DS every year [1]. DS is, therefore, one of the most common genetic conditions in the United Kingdom. DS has a known association with congenital heart problems, gastrointestinal abnormalities, thyroid dysfunction, and also an increased tendency for respiratory infections (RTIs) [2].

RTIs can be split into upper respiratory tract infections (URTIs), and lower respiratory tract infections (LRTIs). Most URTIs are viral in origin and are commonly due to rhinoviruses, coronaviruses, parainfluenza viruses, respiratory syncytial virus (RSV), adenoviruses and influenza viruses. In contrast, the aetiology of LRTIs is more mixed with a study of hospitalised children noting a bacterial (25\%), viral $(25 \%)$ or mixed $(20 \%)$ cause for RTIs [3]. The overall risk of complications is generally low following acute respiratory tract infections [4]. However, the risk of complications is thought to be increased in children with certain comorbidities such as DS.

Important evidence on the burden of respiratory tract infections in children with DS was gathered in Australia. A cohort of 405 children with DS born between 1983 and 1999 was followed up from birth until 2004 [5]. They were hospitalised 3786 times in this period, of which almost one third of admissions was for respiratory tract infections, affecting $52.6 \%$ of all children with DS, with an admission rate of 11.4 per 1,000 person years at risk, a rate 17.9 times higher than in the general paediatric population. Similarly, in a USA study of 217 children with DS born between 1997 and 1999 and followed up until they reached 3 years of age, $42.0 \%$ of hospital admissions were due to RTIs [6].

Despite these findings, data on the comparative frequency of RTIs in children with and without DS are lacking. Uncertainty remains around the burden of RTIs on children with DS in primary care, which has not been studied in detail until now. Families and carers are also lacking vital information regarding the relative risk of re-consultation, and the relative risk of consultation in the presence of certain comorbidities. This study aims to address the evidence gap by undertaking a retrospective cohort study of RTI-related healthcare utilisation in children with DS compared to controls.

\section{Methods}

\section{Objectives}

To quantify healthcare utilisation attributable to RTIs in children with and without DS from 1997 to 2010; to ascertain which children, with and without DS, are most at risk of increased RTI-related healthcare utilisation.

\section{Data sources and definitions}

CALIBER is a database of linked routinely collected electronic health records (EHR) from England [7], comprising data from primary care (Clinical Practice Research Datalink, CPRD) [7], hospital admissions (Hospital Episode Statistics, HES) [7, 8], the Myocardial Ischaemia National Audit Project (MINAP) [9] and the national death registry at the Office for National Statistics (ONS). Read codes assigned by GPs to consultations, or ICD-10 codes assigned to hospitalisations, are used to ascertain the nature of healthcare utilisation.

We developed an algorithm that searched symptom and diagnosis codes, based on reviewing previous code lists used in UK databases for RTIs, and diagnostic and symptom codes were searched through the Read and ICD-10 dictionaries using the R CALIBERcode package [10]. Codes were classified as either "URTI", "LRTI" or "Unclassified RTI" (i.e. uncertainty on whether it was a URTI or LRTI) based on previous code lists and after a consensus meeting including $\mathrm{AS}, \mathrm{ML}, \mathrm{AH}$ and LM. A similar process was undertaken to phenotype DS and comorbidities using any of the Read codes for DS in CPRD and ICD-10 codes for DS in HES. The code lists are available in Online Resource 1.

Within CALIBER, consultation and prescription rates were sourced from CPRD, and hospitalisation rates were sourced from HES. Rates were computed by dividing the number of episodes during the active period in the database by the total number of active person years. Each consultation for an RTI was followed up for up to 28 days or the first hospitalisation for an RTI within 28 days. RTIs were categorised following a ranking system based on RTI-type (LRTI > URTI > unclassified) and setting ( secondary $>$ primary care) if multiple episodes occurred on the same day.

We distinguished four different age groups based on author consensus: infants (0 to 1-year old), toddlers (1-5 years old), juniors (5-10 years old) and young persons (10-18 years old). For admissions lasting greater than 1 day, the length of stay was calculated as "length of stay = discharge date - admission date". For admissions occurring over 1 day, the length of stay was the "length of stay = discharge date - admission date +1 day". 


\section{Participants}

We included all adults and children with DS identified in any of the CALIBER data sources between 1st January 1997 to 25th March 2010 by searching for any of the Read codes for DS in CPRD and by ICD-10 codes in HES recorded as either the primary or secondary discharge diagnosis $(n=3200)$. Individuals with an exit date from the database prior to their entry date to the database (i.e. patient records with data quality issues) were removed $(n=324)$. For each of the remaining adults and children with DS $(n=2876)$, five controls were frequency matched by GP, gender, birth year ( \pm 5 years) and starting date of follow-up. Those who were more than 18 years old at the entry date were subsequently excluded from the study $(n=1884)$.

\section{Sample size and statistical models}

Based on an Australian study of hospitalisations that noted an average of 0.8 and 0.1 RTI-attributable hospital admissions in individuals with and without DS, respectively [5], to identify a difference in hospitalisation rates as large as this between the two groups at $80 \%$ power using a $5 \%$ significance level, 20 individuals per group with the hospitalisation rates above would be required.

Poisson regression was used to calculate consultation, hospitalisation and prescription rates and rate ratios, and their corresponding 95\% confidence intervals. Wald test was used to compare risk of admission following consultation. Due to highly skewed distributions, means and medians were reported, and the Wilcoxon rank-sum test was used to compare length of stay by RTI type, and time-to-hospitalisation.

All data management and analyses were performed using STATA statistical software version 13 and $\mathrm{R}$ version 3.2.3 via the UCL Data Safe Haven.

\section{Study registration}

The protocol for this study was approved by the CPRD-independent scientific advisory committee, reference number 15_041R. The CALIBER record linkage has separate ethical approval (09/H0810/16) for observational clinical research.

\section{Results}

\section{Cohort size, demographics and comorbidities}

This study identified 992 children with DS. They were followed up for a total of 4681 person years at risk, a mean of 4.72 years per child. The 4874 controls were followed up for
Table 1 Demographics and comorbidities of the study population

\begin{tabular}{lll}
\hline & Children with DS & Control \\
\hline Patient & $992(100.0 \%)$ & $4874(100.0 \%)$ \\
Gender & & \\
Male & $528(53.2 \%)$ & $2626(53.9 \%)$ \\
Female & $464(46.8 \%)$ & $2248(46.1 \%)$ \\
Age at entry into cohort & & \\
Infants (0-1 year) & $252(25.4 \%)$ & $1247(25.6 \%)$ \\
Toddlers (1-5 years) & $224(22.6 \%)$ & $1133(23.2 \%)$ \\
Juniors (5-10 years) & $208(21.0 \%)$ & $1044(21.3 \%)$ \\
Young persons (10-18 years) & $308(29.8 \%)$ & $1454(29.8 \%)$ \\
Ethnicity & & \\
Asian or Asian British & $56(3.3 \%)$ & $211(2.5 \%)$ \\
Black or Black British & $48(2.8 \%)$ & $189(2.4 \%)$ \\
Chinese or 'Other' Group & $30(1.7 \%)$ & $114(1.35 \%)$ \\
Mixed & $72(4.2 \%)$ & $393(4.7 \%)$ \\
Unknown & $504(29.5 \%)$ & $4005(47.5 \%)$ \\
White & $1001(58.5 \%)$ & $3523(41.8 \%)$ \\
Comorbidities & & \\
Asthma & $136(13.7 \%)$ & $618(12.7 \%)$ \\
CHD & $393(39.6 \%)$ & $48(1.0 \%)$ \\
Diabetes & $11(1.1 \%)$ & $20(0.4 \%)$ \\
Epilepsy & $18(1.8 \%)$ & $34(0.7 \%)$ \\
Hypothyroidism & $103(10.4 \%)$ & $11(0.2 \%)$ \\
\hline & &
\end{tabular}

a total of 22,837 person years at risk, a mean of 4.69 years per child. Table 1 displays the demographics and comorbidities of the study populations.

\section{Consultation and hospitalisation rates}

RTI-related healthcare utilisation for children with DS is high, and higher than for controls. RTI-related GP consultation rates were 64 per 100 person years for children with DS, and 36 per 100 person years for controls. Corresponding RTI-related hospitalisation rates were 7 per 100 person years for children with DS, and 1 per 100 person years for controls (see Table 2).

When adjusted for age group, children with DS are nearly twice as likely (adjusted relative risk (RR) 1.73; 95\% confidence interval (CI) 1.62-1.84) as matched controls to present to their GP with an RTI in general, and six times more likely (adjusted RR 5.69; 95\% CI 4.82-6.73) to be admitted to hospital with an RTI (see Table 2).

Overall, $74.1 \%$ and $73.4 \%$ of RTI-related hospitalisations occur without any prior GP consultation in for children with DS and controls, respectively.

Approximately $2 \%$ of children with DS are admitted to hospital in the 28 days following a GP consultation for RTI compared to $0.7 \%$ of controls [RR 3.15 (95\% CI 2.35-4.24), unadjusted] (Table 3). 
Table 2 RTI-related GP consultation (top) and hospitalisation (bottom) rates by RTI type in children with DS and controls

\begin{tabular}{|c|c|c|c|c|c|c|c|}
\hline \multirow[t]{2}{*}{ Classification } & \multicolumn{2}{|c|}{ Children with DS } & \multicolumn{2}{|l|}{ Controls } & \multicolumn{3}{|c|}{ Children with DS vs controls } \\
\hline & \# of episodes & $\begin{array}{l}\text { Rate per person } \\
\text { year }[95 \% \mathrm{CI}]\end{array}$ & \# of episodes & $\begin{array}{l}\text { Rate per person } \\
\text { year }[95 \% \mathrm{CI}]\end{array}$ & $\mathrm{RR}[95 \% \mathrm{CI}]$ & $\begin{array}{l}\text { Adjusted RR [95\% } \\
\text { CI] }\end{array}$ & $p$ value \\
\hline \multicolumn{8}{|c|}{ RTI-related GP consultation rates } \\
\hline All & 6013 & $\begin{array}{l}0.638[0.582- \\
0.699]\end{array}$ & 13,957 & $\begin{array}{l}0.363[0.348- \\
0.378]\end{array}$ & $\begin{array}{l}1.760[1.647- \\
1.880]\end{array}$ & $\begin{array}{l}1.726[1.617- \\
1.843]\end{array}$ & $<0.0001$ \\
\hline URTI & 3442 & $\begin{array}{l}0.421[0.385- \\
0.460]\end{array}$ & 9093 & $\begin{array}{l}0.258[0.247- \\
0.270]\end{array}$ & $\begin{array}{l}1.628[1.514- \\
1.750]\end{array}$ & $\begin{array}{l}1.604[1.493- \\
1.723]\end{array}$ & $<0.0001$ \\
\hline LRTI & 874 & $\begin{array}{l}0.119[0.107- \\
0.134]\end{array}$ & 1039 & $\begin{array}{l}0.034[0.031- \\
0.037]\end{array}$ & $\begin{array}{l}3.508[3.108- \\
3.955]\end{array}$ & $\begin{array}{l}3.589[3.188- \\
4.041]\end{array}$ & $<0.0001$ \\
\hline Unclassified RTI & 1697 & $\begin{array}{l}0.199[0.179- \\
0.220]\end{array}$ & 3825 & $\begin{array}{l}0.114[0.109- \\
0.120]\end{array}$ & $\begin{array}{l}1.739[1.588- \\
1.902]\end{array}$ & $\begin{array}{l}1.759[1.609- \\
1.923]\end{array}$ & $<0.0001$ \\
\hline \multicolumn{8}{|c|}{ RTI-related hospitalisation rates } \\
\hline All & 473 & $\begin{array}{l}0.067[0.058- \\
0.077]\end{array}$ & 327 & $\begin{array}{l}0.013[0.011- \\
0.014]\end{array}$ & $\begin{array}{l}5.342[4.506- \\
6.332]\end{array}$ & $\begin{array}{l}5.693[4.818- \\
6.727]\end{array}$ & $<0.0001$ \\
\hline URTI & 205 & $\begin{array}{l}0.035[0.030- \\
0.043]\end{array}$ & 187 & $\begin{array}{l}0.007[0.006- \\
0.009]\end{array}$ & $\begin{array}{l}4.814[3.842- \\
6.029]\end{array}$ & $\begin{array}{l}4.989 \text { [4.007- } \\
6.211]\end{array}$ & $<0.0001$ \\
\hline LRTI & 213 & $\begin{array}{l}0.032[0.026- \\
0.038]\end{array}$ & 73 & $\begin{array}{l}0.003[0.002- \\
0.004]\end{array}$ & $\begin{array}{l}10.557[7.847- \\
14.321]\end{array}$ & $\begin{array}{l}11.295[8.448- \\
15.101]\end{array}$ & $<0.0001$ \\
\hline Unclassified RTI & 55 & $\begin{array}{l}0.010[0.007- \\
0.013]\end{array}$ & 67 & $\begin{array}{l}0.003[0.002- \\
0.004]\end{array}$ & $\begin{array}{l}3.468[2.314- \\
5.158]\end{array}$ & $\begin{array}{l}3.578[2.444- \\
5.239]\end{array}$ & $<0.0001$ \\
\hline
\end{tabular}

Table 3 Risk of RTI-related hospitalisation following an RTI-related GP consultation within 28 days in children with DS and controls

\begin{tabular}{|c|c|c|c|c|c|c|c|c|}
\hline \multirow[t]{2}{*}{ Classification } & \multicolumn{3}{|c|}{ Children with DS } & \multicolumn{3}{|l|}{ Controls } & \multicolumn{2}{|c|}{ Children with DS vs Controls } \\
\hline & $\begin{array}{l}\text { \# of } \\
\text { consul- } \\
\text { tations }\end{array}$ & $\begin{array}{l}\text { \# of } \\
\text { hospitali- } \\
\text { sations }\end{array}$ & $\operatorname{Risk}[95 \% \mathrm{CI}]$ & \# of consultations & $\begin{array}{l}\text { \# of } \\
\text { hospitali- } \\
\text { sations }\end{array}$ & $\operatorname{Risk}[95 \% \mathrm{CI}]$ & $\begin{array}{l}\text { Risk ratio }[95 \% \\
\mathrm{CI}]\end{array}$ & $p$ value \\
\hline All & 4685 & 97 & $\begin{array}{l}0.021[0.017- \\
0.025]\end{array}$ & 11,877 & 78 & $\begin{array}{l}0.007[0.005- \\
0.008]\end{array}$ & $\begin{array}{l}3.153[2.345- \\
4.239]\end{array}$ & $<0.0001$ \\
\hline URTI & 2769 & 42 & $\begin{array}{l}0.015[0.011- \\
0.021]\end{array}$ & 7915 & 43 & $\begin{array}{l}0.005[0.004- \\
0.007]\end{array}$ & $\begin{array}{l}2.792[1.829- \\
4.262]\end{array}$ & $<0.0001$ \\
\hline LRTI & 621 & 15 & $\begin{array}{l}0.024[0.014- \\
0.040]\end{array}$ & 838 & 7 & $\begin{array}{l}0.008[0.003- \\
0.017]\end{array}$ & $\begin{array}{l}2.892[1.186- \\
\quad 7.050]\end{array}$ & 0.0168 \\
\hline Unclassified RTI & 1295 & 40 & $\begin{array}{l}0.031[0.022- \\
0.042]\end{array}$ & 3124 & 28 & $\begin{array}{l}0.009[0.006- \\
0.013]\end{array}$ & $\begin{array}{l}3.446[2.135- \\
5.561]\end{array}$ & $<0.0001$ \\
\hline
\end{tabular}

Time to RTI-related hospitalisation following an RTIrelated GP consultation is the same in both groups, with a median of 8.0 days (95\% CI 3.0-19.0) in children with DS and 8.0 days (95\% CI 2.0-18.0) in matched controls. However, children with DS are more likely (OR 1.69; $95 \%$ CI 1.57-1.82) to re-consult with their GP for an RTI within 28 days of a prior RTI-related consultation compared to matched controls, with $24.3 \%$ children with DS re-consulting compared to $16.0 \%$ of matched controls.

Over the study period between 1997 and 2010, RTIrelated GP consultation and hospitalisation rates were consistently higher in children with DS compared to controls. The disparities increased over time; in 1999, children with DS had a third more RTI-related consultations, compared to $80 \%$ more in 2009 . An increase was similarly noted for RTI-related hospitalisations. This is shown in Fig. 1.

\section{Healthcare utilisation by type of RTI}

Across all RTI types (i.e. LRTI, URTI, and unclassified RTI), children with DS consistently consult GPs and are hospitalised for RTIs more frequently compared to controls. The differences between children with DS and controls were most pronounced for LRTIs in GP consultations (RR 3.59; 95\% CI 3.19-4.04) and hospitalisations (RR $11.30 ; 95 \%$ CI 8.45-15.10). This is illustrated in Table 2. 


\section{Length of hospitalisations for RTIs}

Overall, as shown in Table 4, the length of stay in hospital due to RTIs is significantly longer for children with DS (mean 5.2; 95\% CI 5.0-5.4 days per admission) compared to controls (mean 2.4; 95\% CI 2.2-2.6, $p<0.0001$ ). In keeping with LRTIs being more severe than URTIs or unclassified RTIs, LRTI-related hospitalisations last longer for children with DS (mean 7.8; 95\% CI 7.4-8.1 days per admission) and controls (mean 4.2; 95\% CI 3.8-4.7).
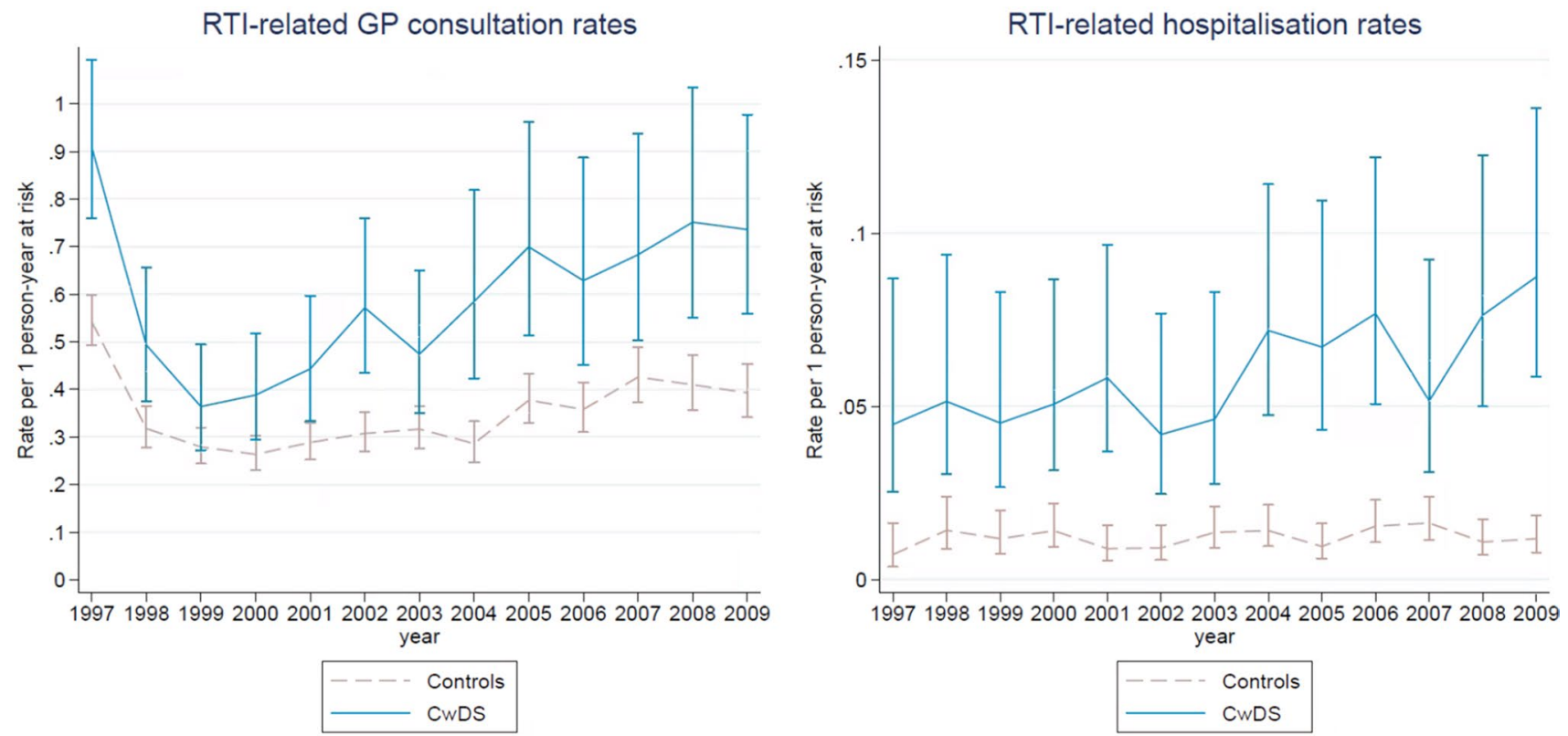

Fig. 1 Annual RTI-related GP consultation and hospitalisation rates in children with DS compared to controls

Table 4 Length of stay in hospital for LRTI, URTI and unclassified RTI in days in children with DS and controls

\begin{tabular}{|c|c|c|c|c|c|}
\hline \multirow[t]{2}{*}{ Classification } & \multicolumn{2}{|c|}{ Children with DS } & \multicolumn{2}{|l|}{ Controls } & \multirow[t]{2}{*}{$p$ value } \\
\hline & Mean $[95 \% \mathrm{CI}]$ & Median [IQR] & Mean $[95 \% \mathrm{CI}]$ & Median [IQR] & \\
\hline All & $5.2[5.0-5.4]$ & $2.0[1.0-5.0]$ & $2.4[2.2-2.6]$ & $2.0[1.0-2.0]$ & $<0.0001$ \\
\hline URTI & $2.4[2.2-2.6]$ & $2.0[1.0-2.0]$ & $1.9[1.7-2.1]$ & $1.0[1.0-2.0]$ & 0.0325 \\
\hline LRTI & $7.8[7.4-8.1]$ & $5.0[3.0-10.0]$ & $4.2[3.8-4.7]$ & $3.0[2.0-5.0]$ & $<0.0001$ \\
\hline Unclassified RTI & $5.6[5.0-6.3]$ & $2.0[1.0-4.0]$ & $1.9[1.6-2.3]$ & $1.0[1.0-2.0]$ & 0.0265 \\
\hline
\end{tabular}

Table 5 RTI-related GP consultation (top) and hospitalisation (bottom) rates stratified by age groups and compared between children with DS and controls

\begin{tabular}{|c|c|c|c|c|c|c|}
\hline \multirow[t]{2}{*}{ Classification } & \multicolumn{2}{|c|}{ Children with DS } & \multicolumn{2}{|l|}{ Controls } & \multicolumn{2}{|c|}{ Children with DS vs controls } \\
\hline & \# of episodes & Rate per person year $[95 \% \mathrm{CI}]$ & \# of episodes & Rate per person year $[95 \% \mathrm{CI}]$ & $\mathrm{RR}[95 \% \mathrm{CI}]$ & $p$ value \\
\hline \multicolumn{7}{|c|}{ RTI-related GP consultation rates } \\
\hline Infants & 270 & $1.495[1.252-1.790]$ & 961 & $1.236[1.140-1.341]$ & $1.210[0.975-1.490]$ & 0.0371 \\
\hline Toddlers & 2387 & $1.225[1.052-1.422]$ & 6185 & $0.813[0.761-0.867]$ & 1.508 [1.339-1.695] & $<0.0001$ \\
\hline Juniors & 1727 & $0.715[0.621-0.822]$ & 3365 & $0.324[0.302-0.346]$ & $2.211[1.946-2.505]$ & $<0.0001$ \\
\hline Young person & 1629 & $0.364[0.319-0.414]$ & 3446 & $0.195[0.184-0.208]$ & $1.861[1.648-2.096]$ & $<0.0001$ \\
\hline \multicolumn{7}{|c|}{ RTI-related hospitalisation rates } \\
\hline Infants & 73 & $0.515[0.388-0.695]$ & 56 & $0.079[0.059-0.109]$ & 6.491 [4.190-10.087] & $<0.0001$ \\
\hline Toddlers & 240 & $0.140[0.116-0.171]$ & 176 & $0.031[0.026-0.036]$ & $4.582[3.554-5.893]$ & $<0.0001$ \\
\hline Juniors & 93 & $0.058[0.046-0.075]$ & 56 & $0.008[0.006-0.011]$ & $7.330[5.002-10.792]$ & $<0.0001$ \\
\hline Young person & 67 & $0.024[0.018-0.032]$ & 39 & $0.004[0.003-0.005]$ & 6.669 [4.296-10.432] & $<0.0001$ \\
\hline
\end{tabular}




\section{Healthcare utilisation stratified by age, gender and comorbidities}

Consultation and hospitalisation rates for RTIs were higher in children with DS compared to controls across all age groups. This difference was particularly pronounced for hospitalisations (see Table 5).

There is no significant gender-based difference for RTIrelated GP consultation rates for children with DS (adjusted RR 1.08; 95\% CI 0.96-1.21) and matched controls (adjusted RR 0.99; 95\% CI 0.93-1.05). Girls have lower RTI-related hospitalisation rates compared to boys in both children with DS (adjusted RR 0.70; 95\% CI 0.55-0.89) and matched controls (adjusted RR 0.69; 95\% CI 0.54-0.88).

Amongst children with DS, those who have congenital heart disease (CHD) have increased RTI-related consultation (adjusted RR 1.21; 95\% CI 1.04-1.40) and hospitalisation rates (adjusted RR 3.07; 95\% CI 2.38-3.95) compared to those without CHD. A similar pattern is observed in controls for RTI-related consultations (adjusted RR 1.63; 95\% 1.00-2.67) and hospitalisations (adjusted RR 3.89; 95\% CI 1.25-12.11).

Children with DS with asthma attend their GP for RTIrelated consultations more often compared to children with DS without asthma (adjusted RR 2.06; 95\% CI 1.74-2.44) and are hospitalised more often (adjusted RR 1.68; 95\% CI 1.23-2.30). Controls with asthma attend their GP more often (adjusted RR 1.75; 95\% CI 1.61-1.91) and are hospitalised more often (adjusted RR 2.65; 95\% CI 2.01-3.50) for RTIs relative to controls without asthma.

\section{Antibiotic prescribing}

Children with DS are prescribed twice as many antibiotics compared to matched controls, with a rate of 77 per 100 person years, compared to 32 per 100 person years for controls (adjusted RR 2.34; 95\% CI 2.19-2.49). When restricted to antibiotic prescriptions prescribed on the same day as an RTI-related GP consultation, children with DS are twice as likely to be prescribed an antibiotic for an RTI compared to matched controls, with a rate of 42 per 100 person years for children with DS compared to 19 per 100 person years for controls (adjusted RR 2.26; 95\% CI 2.10-2.43).

When stratified by RTI type, children with DS receive significantly more antibiotics on the same day as an RTI-related GP consultations when presenting with URTIs (57.0\%; 95\% CI $55.3-58.6 \%$ vs $47.9 \%$; $95 \%$ CI $46.9-49.0 \%$ ) and unclassified RTIs (52.9\%; 95\% CI 50.5-55.3\% vs $32.0 \%$; $95 \%$ CI $30.5-33.5 \%)$. There is no significant difference in the high proportion of LRTIs that are prescribed antibiotics in children with DS compared with controls (87.0\% vs. $82.5 \%)$.

When stratified by age group, infants with DS have the highest rate of RTI-related antibiotic prescribing compared to all other groups including controls, with rates of 80 per 100 person years (95\% CI 65-99). Prescribing rates are significantly higher for each age group in DS compared to the equivalent age group amongst controls.

\section{Discussion}

This study demonstrates that RTIs in children with DS lead to a significant number of presentations to GPs and to hospitals every year, posing a significant burden on patients and their families. Compared to controls, children with DS attend GP consultations for RTIs almost twice as often, are hospitalised six times as often, stay in hospital longer, and are prescribed antibiotics more frequently. The presence of comorbidities increased RTI-related healthcare utilisation for both groups. Children with DS and controls were hospitalised and attended GPs for RTIs less often as they aged.

Children with DS were three times more likely to be admitted to hospital for an RTI following an RTI-related GP consultation than controls, with a risk of $2.1 \%$, compared to $0.7 \%$ amongst controls. The finding of $0.7 \%$ in controls $(95 \%$ CI $0.5-0.8 \%$ ) is similar to a recent UK population based cohort study which noted a baseline risk of $0.9 \%$ (95\% CI $0.7-1.2 \%)$ [11].

Notably, for both children with DS and controls, more than $70 \%$ of RTI-related hospitalisations were not preceded by a recorded GP consultation.

There are number of limitations to our study, described below.

\section{Strengths and limitations}

This is the largest study of RTI-related healthcare utilisation in children with DS worldwide $(n=992)$ and provides novel insights as the first study known to us to assess healthcare utilisation and antibiotic prescribing in primary care for children with DS, as well as to compare them with controls. It provides valuable data on the prevalence of antibiotic prescribing, and the associated impact of key comorbidities. Comorbidity prevalence figures in this study are similar to those in existing literature. The calculated prevalence of CHD in DS of $39.6 \%$ compares to rates of $33.7-58.2 \%$ in other studies [12-15]. A prevalence of $13.7 \%$ for asthma compares to rates of $3.1-19.4 \%[16,17]$.

There are number of limitations to this study which should be noted when considering our findings. By relying on READ and ICD-10 codes for diagnoses, this study may be subject to misclassification bias. It is known that considerable inter-practice variation exists in coding certain conditions such as RTIs. For example, READ or ICD-10 codes for "respiratory tract infection" could be either an URTI 
or LRTI. We aimed to address this by separately considering unclassified RTI types, but misclassification bias may remain. Misclassification may particularly be present for asthma, which is not typically diagnosed in the under $5 \mathrm{~s}$ in the UK. Individual episodes could have been missed through non-recording by GPs, or use of free text entries, although it is unknown whether this would differ between children with DS and controls. An additional proportion of medically attended RTIs in both children with DS and controls will be missed, as RTIs are seen not only by GPs but also at other ambulatory care centres (i.e. urgent care centres, outof-hours GP) and A\&E. Finally, and importantly, research has noted that most RTIs do not lead to a GP consultation [18]. There is little qualitative evidence on healthcare seeking behaviour in families of children with DS, and whether this varies from other patient groups. Until such evidence can be gathered, we cannot know whether the data partially reflects a difference in propensity to consult when children with DS and controls have RTIs, rather than a true difference in RTI incidence.

\section{Implications for clinical practice and research}

Healthcare professionals in primary care should be vigilant when assessing children with DS with the knowledge that subsequent hospital admissions are more likely. Morphological and functional anomalies of the airway causing (partial) obstruction, particularly midfacial hypoplasia and airway malacia [19], alongside immunological variations including reduced leukocytes, particularly T- and B-cell subpopulations [20-23], increase susceptibility to and severity of respiratory infections. Increased admissions may also be due to uncertainty regarding speed of deterioration or oxygen requirements, and many admissions may be precautionary, also reflected by the higher prescribing rates in children with DS. In this context, parents have reported that some doctors seem 'afraid' of children with DS [24]. It will be important to identify whether the high rates of admission are related to severity of the infection, time at presentation, time and type of antibiotics prescribed, or other factors. Further research should determine why more than $70 \%$ of RTI-related hospitalisations were not preceded by a recorded GP consultation as this may represent an opportunity to avert admission through timely community-based treatment. Regional analyses may be helpful, comparing Clinical Commissioning Groups with high and low rates of RTI-related health utilisation, to highlight areas of best practice and whether local community initiatives exist that can avert unnecessary attendances.

This study also found that certain subgroups are at greater risk of hospitalisation. It may be that those at risk of RTI-related hospitalisation could be defined in greater detail, thus enabling the development of a symptom-based scoring algorithm modelled on algorithms that already exist for many other conditions [11, 25, 26]. However, symptoms are not well recorded in routine records. Although not explored by this study, further research may also want to examine prescribing rates for antivirals for influenza in children with DS. These are rarely prescribed for children without DS, despite recommendations to do so $[27,28]$. Regarding public health recommendations, this study lends weight to the recommendation by the Down's Syndrome Medical Interest Group that children with DS should be considered for annual influenza vaccination at all ages [29], particularly in infancy.

\section{Conclusion}

This is the first study of RTI-related healthcare utilisation in children with DS compared to controls utilising linked primary and secondary care data. We show that healthcare utilisation is high in this population and higher than controls. Further research is recommended, carefully quantifying healthcare behaviours and health professional decisionmaking, to optimise care for this at-risk group.

Author Contribution LM, AS, ML, PL and AH contributed to the study conception and design, and to acquisition, analysis and interpretation along with EA. Material preparation, data collection and analysis were predominantly performed by LM with advice from all other authors. The first draft of the manuscript was written by LM and EA and all authors commented on subsequent versions of the manuscript. All authors read and approved the final manuscript.

\section{Compliance with ethical standards}

Conflict of interest This study was completed as part of LM's National Institute for Health Research (NIHR) Doctoral Research Fellowship (DRF-2014-07-005) hosted at the UCL GOS Institute of Child Health. Whilst preparing the fellowship application, LM was in receipt of a NIHR Academic Clinical Fellowship in Public Health at King's College London. CALIBER is funded by a Wellcome Trust project grant (086091/Z/08/Z) and a NIHR programme grant (RP-PG-0407-10314). AS is supported by the National Institute of Health Research University College London Hospitals Biomedical Research Centre. This study was supported by the UCL Institute of Health Informatics Research by providing both infrastructure, access and technical support in utilising CALIBER. AH is an NIHR senior investigator. PL is supported by the National Institute for Health Research (NIHR) Applied Research Collaboration (ARC) South London at King's College Hospital NHS Foundation Trust. The views expressed are those of the author(s) and not necessarily those of the NHS, the NIHR or the Department of Health and Social Care.

Ethics approval The protocol for this study was approved by the CPRD-independent scientific advisory committee, reference number 15_041R. The CALIBER record linkage has separate ethical approval (09/H0810/16) for observational clinical research. This study was, therefore, performed in accordance with the ethical standards laid down in the 1964 Declaration of Helsinki and its later amendments. 
Open Access This article is licensed under a Creative Commons Attribution 4.0 International License, which permits use, sharing, adaptation, distribution and reproduction in any medium or format, as long as you give appropriate credit to the original author(s) and the source, provide a link to the Creative Commons licence, and indicate if changes were made. The images or other third party material in this article are included in the article's Creative Commons licence, unless indicated otherwise in a credit line to the material. If material is not included in the article's Creative Commons licence and your intended use is not permitted by statutory regulation or exceeds the permitted use, you will need to obtain permission directly from the copyright holder. To view a copy of this licence, visit http://creativecommons.org/licenses/by/4.0/.

\section{References}

1. Wu J, Morris JK. Trends in maternal age distribution and the live birth prevalence of Down's syndrome in England and Wales: 1938-2010. Eur J Hum Genet. 2013;21:943-7.

2. Watts R, Vyas H. An overview of respiratory problems in children with Down's syndrome. Arch Dis Childh. 2013;812-817.

3. Korppi M, Heiskanen-Kosma T, Jalonen E, Saikku P, Leinonen M, Halonen P, Makela PH. Aetiology of community-acquired pneumonia in children treated in hospital. Eur $\mathrm{J}$ Pediatr. 1993;152:24-30.

4. Petersen I, Johnson A, Islam A, Duckworth G, Livermore D, Hayward A. Protective effect of antibiotics against serious complications of common respiratory tract infections: retrospective cohort study with the UK General Practice Research Database. BMJ. 2007;335:982.

5. Fitzgerald P, Leonard H, Pikora T, Bourke J, Hammond G. Hospital admissions in children with down syndrome: experience of a population-based cohort followed from birth. PLoS ONE. 2013;8:e70401.

6. So SA, Urbano RC, Hodapp RM. Hospitalizations of infants and young children with Down syndrome: evidence from inpatient person-records from a statewide administrative database. J Intellect Disabil Res. 2007;51:1030-8.

7. Herrett E, Gallagher AM, Bhaskaran K, Forbes H, Mathur R, van Staa T, Smeeth L. Data resource profile: clinical practice research datalink (CPRD). Int J Epidemiol. 2015;44:827-36.

8. Hospital Episode Statistics [https://content.digital.nhs.uk/hes]

9. Herrett E, Smeeth L, Walker L, Weston C, Group MA. The myocardial ischaemia national audit project (MINAP). Heart. 2010;96:1264-7.

10. CALIBERcodelist [https://caliberanalysis.r-forge.r-project.org/]

11. Hay AD, Redmond NM, Turnbull S, Christensen H, Thornton H, Little P, Thompson M, Delaney B, Lovering AM, Muir P et al: Development and internal validation of a clinical rule to improve antibiotic use in children presenting to primary care with acute respiratory tract infection and cough: a prognostic cohort study. Lancet Respir Med. 2016.

12. Halliday J, Collins V, Riley M, Youssef D, Muggli E. Has prenatal screening influenced the prevalence of comorbidities associated with Down syndrome and subsequent survival rates? Pediatrics. 2009;123:256-61.
13. Elmagrpy Z, Rayani A, Shah A, Habas E, Aburawi EH. Down syndrome and congenital heart disease: why the regional difference as observed in the Libyan experience? Cardiovasc J Afr. 2011;22:306-9.

14. Bergstrom S, Carr H, Petersson G, Stephansson O, Bonamy AK, Dahlstrom A, Halvorsen CP, Johansson S. Trends in congenital heart defects in infants with down syndrome. Pediatrics 2016;138.

15. de Rubens FJ, del Pozzo MB, Pablos Hach JL, Calderón Jiménez C, Castrejón Urbina R. Heart malformations in children with down syndrome. Revista Española de Cardiologia. 2003;56:894-9.

16. Muñoz-López F. Pediatrics, Down's syndrome and allergic disease. Int Med Rev Down Syndr. 2011;15:8-13.

17. Weijerman ME, Brand PL, van Furth MA, Broers CJ, Gemke RJ. Recurrent wheeze in children with down syndrome: is it asthma? Acta Paediatr. 2011;100:e194-e197197.

18. Little P, Stuart B, Andreou P, McDermott L, Joseph J, Mullee M, Moore M, Broomfield S, Thomas T, Yardley L. Primary care randomised controlled trial of a tailored interactive website for the self-management of respiratory infections (Internet Doctor). BMJ open. 2016;6:e009769.

19. Tutor JD, Gosa MM. Dysphagia and aspiration in children. Pediatr Pulmonol. 2012;47:321-37.

20. Ram G, Chinen J. Infections and immunodeficiency in Down syndrome. Clin Exp Immunol. 2011;164:9-16.

21. Bloemers BL, Broers CJ, Bont L, Weijerman ME, Gemke RJ, van Furth A. Increased risk of respiratory tract infections in children with Down syndrome: the consequence of an altered immune system. Microb ad Infect. 2010;12:799-808.

22. Kusters MAA, Verstegen RHJ, Gemen EFA, De Vries E. Intrinsic defect of the immune system in children with Down syndrome: a review. Clin Exp Immunol. 2009;156:189-93.

23. Bloemers BL, van Bleek GM, Kimpen JL, Bont L. Distinct abnormalities in the innate immune system of children with Down syndrome. J Pediatr. 2010;156:804-9.

24. Minnes P, Steiner K. Parent views on enhancing the quality of health care for their children with fragile $\mathrm{X}$ syndrome, autism or Down syndrome. Child Care Health Dev. 2009;35:250-6.

25. Tomita K, Sano H, Chiba Y, Sato R, Sano A, Nishiyama O, Iwanaga T, Higashimoto Y, Haraguchi R, Tohda Y. A scoring algorithm for predicting the presence of adult asthma: a prospective derivation study. Prim Care Respir J. 2013;22:51-8.

26. Hay AD, Birnie K, Busby J, Delaney B, Downing H, Dudley J, Durbaba S, Fletcher M, Harman K, Hollingworth W, et al. The diagnosis of urinary tract infection in young children (DUTY): a diagnostic prospective observational study to derive and validate a clinical algorithm for the diagnosis of urinary tract infection in children presenting to primary care with an acute illness. Health Technol Assess. 2016;20:1-294.

27. NICE: Amantadine, oseltamivir and zanamivir for the treatment of influenza. In: Technology appraisal guidance [TA168]. 2009.

28. Public Health England: PHE guidance on use of antivirals for the treatment and prophylaxis of influenza. In: Pandemic flu: public health response. 2014.

29. Marder L, Down's syndrome medical interest group: immunisation: protecting people with Down's syndrome against infection. In: 2014. 